\title{
Characterization of a prototype imaging calorimeter for the Advanced Particle-astrophysics Telescope from an Antarctic balloon flight and CERN beam test
}

\author{
Zachary Hughes $^{a, *}$ on behalf of the APT Collaboration \\ (a complete list of authors can be found at the end of the proceedings) \\ E-mail: zdhughes@wustl.edu
}

We report the results and analysis methods from field-testing the imaging calorimeter prototype for the Advanced Particle-astrophysics Telescope (APT) through an antarctic balloon flight (in the 2019 austral Antarctic balloon season) and through a CERN heavy-ion beam test in 2018. The Advanced Particle-astrophysics Telescope is a proposed space-based gamma- and cosmic-ray instrument that utilizes a novel distributed imaging calorimeter for both particle tracking and energy reconstruction. The imaging CsI calorimeter (ICC) consists of a CsI:Na scintillator read out by (WLS) fibers in both the $\mathrm{x}$ - and y-planes. To function both as a gamma-ray and cosmic-ray instrument APT must operate over a large dynamic range, from the single photon-election regime for low energy gamma-ray events to high- $Z$ cosmic-ray events. Analysis of data from a 150 $\mathrm{mm}$ x $150 \mathrm{~mm}$ prototype instrument (APTlite) on a piggy-back flight on the 2019 SuperTIGER2.3 balloon instrument provided cosmic ray data that were used to demonstrate the key detector and electronics elements of the ICC. Significantly, analysis of flight data demonstrated the large dynamic range of the instrument, showing the possibility to reconstruct the nuclear charge through analysis of the scintillation tail of saturating high-Z cosmic-ray events by utilizing the deep memory depth available to the TARGET waveform digitizer electronics. Spatial reconstruction of events was performed using a two-sided Voigt profile demonstrating position localization within the imaging calorimeter plane to less 3 WLS fiber widths. Charge resolution was evaluated on a 50 $\mathrm{mm} \times 50 \mathrm{~mm}$ prototype placed in the $150 \mathrm{GeV} / \mathrm{nuc}, \mathrm{A} / \mathrm{Z}=2.2 \mathrm{CERN}$ SPS beam line. Nuclei were tagged using HNX/TIGERISS silicon-strip detectors and silicon pad detectors, which allowed for fragmentation cuts in the data. The vastly saturating signals were reconstructed from the CsI:Na scintillation tail and show an APT charge resolution up to $Z=11$ (with experimental limitations preventing full evaluation for $Z$ larger 11) and demonstrated no significant non-linearity in the $Z^{2}$ measurement derived from the CsI:Na optical signal response up to $Z=82$.

$37^{\text {th }}$ International Cosmic Ray Conference (ICRC 2021)

July 12th-23rd, 2021

Online - Berlin, Germany

\footnotetext{
${ }^{*}$ Presenter
} 


\section{Overview}

The Advanced Particle-astrophysics Telescope (APT) is a proposed probe-class gamma-ray and cosmic-ray mission aiming to provide an order of magnitude increase in sensitivity over FermiLAT as well as a Compton-imaging mode to cover $\mathrm{MeV}$ to $\mathrm{TeV}$ gamma-ray energies. A key enabling technology for maximizing the instrument's effective area is the imaging CsI calorimeter (ICC). The ICC is a dispersed imaging calorimeter consisting of layers of sodium-doped cesium iodide scintillator (CsI:Na) read-out by crossed wavelength shifting (WLS) fibers. These layers are located evenly throughout the instrument and replace the typical calorimeter-on-the-bottom design and use of passive converter layers common to gamma-ray detectors used in the pair-production regime. The evenly distributed ICC layers in APT allows for up-down instrument viewing symmetry and an instantaneous field of view twice that of the Fermi-LAT. The WLS fibers allow for a large instrument cross-section by piping the CsI:Na scintillation light to the edge of the detector to be read out by low-voltage silicon photomultipliers (SiPMs). This design reduces the need for electronics and other passive material within the detector volume and reduces instrument cost and complexity. The basic detector geometry is shown in Fig. 1 and described in more detail in another paper [1]

Fig. 1 shows a cross-section of an ICC layer. Scintillation light that is not totally internally reflected is absorbed and isotropically re-emitted by the WLS fibers. Light which is re-emitted within the fibers' cladding-core critical angle is piped to the edge of the detector and read out by SiPMs. Because light-piping relies on total internal reflection (TIR), the arriving light at the SiPMs is only a small fraction of the initial scintillation light. To help compensate for this, a novel dual core WLS fiber is employed in the current ICC prototypes. A secondary red core above the crystal-adjacent green core captures escaping light from the green fiber and provides a second opportunity for piping to the SiPMs.

Over the course of development, multiple iterations of the ICC have been constructed for evaluation. The first generation prototypes consist of a $50 \times 50 \times 5 \mathrm{~mm}$ CsI:Na crystal and a single plane of
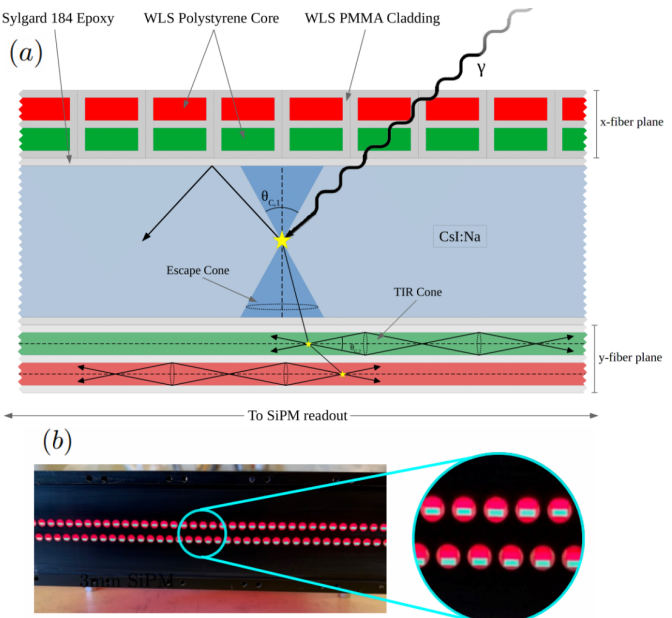

Figure 1: (a) Cross-sectional diagram of the APT imaging CsI calorimeter. Adapted from [2]. (b) Photograph of the far ends of the WLS fibers which are read out by SiPMs. either green or red-green $2 \mathrm{~mm}$ WLS fibers. A red-green prototype was evaluated in 2018 at CERN, in a heavy-ion beam. A second generation prototype was made for flight on SuperTIGER-2.3 in 2019. This detector consists of a $150 \times 150 \times 5 \mathrm{~mm}$ CsI:Na crystal read out in both planes by red-green WLS fibers. This allowed for evaluation of the ICC event localization using CRs detected in coincidence with SuperTIGER (ST). A new generation of ICC planes are under construction for planned beam tests and an Antarctic balloon flight [1] 


\section{CERN Beam Test}

An APT ICC prototype was placed in the CERN test beam (H8A), in the North Area SPS beam line for a two week run in November-December 2018. The prototype was placed in the beam during a scheduled heavy-ion run originally planned for the evaluation of silicon strip detectors (SSDs) designed for the Heavy Nuclei eXplorer (HNX) and in a SuperTIGER upgrade[3]. The beam was a $150 \mathrm{GeV} /$ nuc lead primary and fragmented beam $(\mathrm{A} / \mathrm{Z}=2.4,2.2$, 2.0). During this experiment, the detector line was

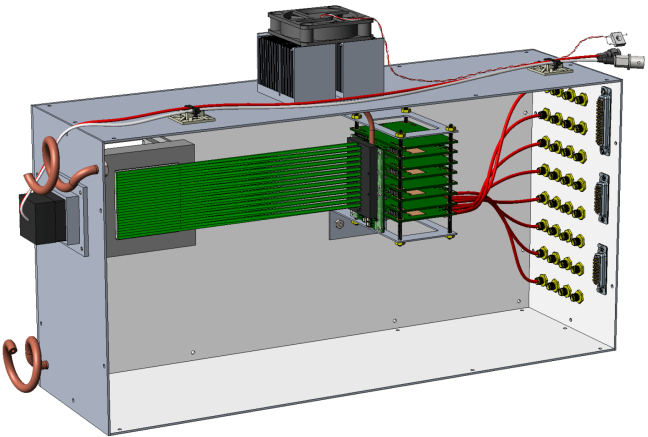

Figure 2: Rendering of the detector box used in the CERN beam test. configured such that the APT prototype was located between two HNX SSDs and 4 large area pad detectors, allowing for the tagging and identification of non-fragmenting events from helium $(\mathrm{Z}=$ $2)$ to lead $(Z=82)$. Figure 3 shows the prototype's position relative to the other experiments in the beam line during this time. For this configuration the beam was fragmented with a mass-to-charge ratio of $\mathrm{A} / \mathrm{Z}=2.2$.

The detector used for the CERN run (shown in Figure 2) consisted of a $50 \times 50 \times 5 \mathrm{~mm}$ CsI:Na crystal with a single plane of $252 \mathrm{~mm}$ red-green WLS fibers. The crystal sat within an aluminum housing with an open bottom, allowing for the capture of both direct scintillation light from the crystal and the piped signal at the ends of the fiber. The $13 \mathrm{~cm}$ WLS fibers' ends were bonded to the crystal with SYLGARD 186 silicone epoxy and read out with Hamamatsu $3 \mathrm{~mm}$ S14160$3050 \mathrm{SiPMs}$, while the integrated scintillation signal from the bare crystal was captured with a Hamamatsu R1398HA photomultiplier tube (PMT). To integrate the scintillation light from the open side of the CsI crystal, a diffuse-reflective Tyvek housing was constructed around the aluminum holder. The readout electronics consisted of a first prototype of our discrete pre-amplifier boards as well as SiPM bias voltage control via board utlizing AD5504 4-channel 12bit high-voltage digital-to-analog (DAC) ICs. The preamps were based on a design incorporating a DC-coupled transimpedance amplifier front end, with high-side bias voltage control, followed by a two stage pole-zero compensating shaper to provide unipolar single p.e. pulses with a width of about 10 nsec. While the preamp gain was fixed, the digital bias-voltage control allows for tuning the signal amplitudes to levels suitable for the read-out electronics without a dramatic change in quantumefficiency. Due to extremely large amplitude signals generated by the heavy nuclei, the voltage was set just above the SiPMs' $38 \mathrm{~V}$ breakdown voltage to $38.5 \mathrm{~V}$.

Since the CERN run had been scheduled to occur just months after our first APT R\&D program was funded, and the beam line would not be available for several more years, we quickly assembled a minimal detector system to take advantage of the heavy ion beam. Since the Target $C$ based readout was not ready at the time of the beam test and our primary objective was to determine the saturation

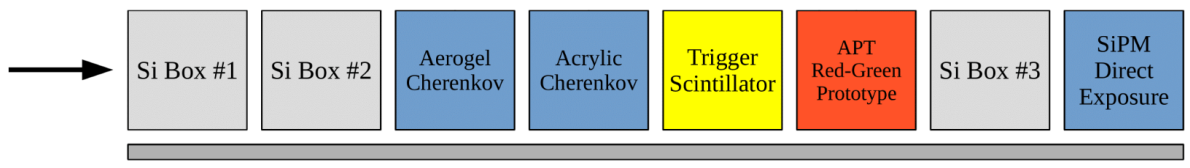

Figure 3: The APT prototype detector box location in the CERN beam line (red). Adapted from [2]. 
behaviour of the CsI:Na+WLS-fiber detector at very high $Z$, we made use of available equipment to instrument the run. To this end, we used spare VERITAS Flash Analog to Digital Converter (FADC) boards in place of the Target ASICs to provide waveform digitization of the SiPM signals. While designed for use in imaging atmospheric Cherenkov telescopes (IACT), the VERITAS readout electronics had a number of features well suited to use in the CERN beam test. Its $500 \mathrm{MSa} / \mathrm{s}$ sampling rate is fast enough to resolve individual photo-electrons while its $8 \mu$ s memory depth was capable of capturing much of the several- $\mu$ sec long tail of the CsI:Na pulse. However, challenges were present in using the VERITAS FADC system. First, a shared event number was not present between the FADC system and the rest of detectors in the beam line and only software time-tagging could be used to match events. Second, the VERITAS FADC system employs a gain-switching mechanism to increase its dynamic range. Because the CsI:Na scintillation pulses from heavy ions can be orders of magnitude larger and longer in duration than the Cherenkov pulses for which the system was designed, analysis of the CERN beam waveform data was challenging and some data and gain-settings proved unusable for our analysis.

\subsection{Beam test analysis \& results}

The VERITAS FADC system uses a highlow gain-switching mechanism (HILO) to increase its dynamic range. When a signal is detected above the system's ADC range an ana$\log$ switch is thrown and a delayed, unity-gain signal is recorded instead. Because of the fast rise time of the scintillation pulse, this switching resulted in distortions around the leading edge of the signal waveform, making it necessary to exclude the region around the switching time. The HILO switch also automatically resets after a fixed time of about 300 nsec. While this is larger than the duration of atmospheric Cherenkov pulses, this behavior produced additional challenges in fitting to the time profile of the pulses. Fig. 4a shows the result of this HILO gain-switching. To correct for this, a non-parametric pulse profile was found using the non-switched waveform data using principal component analysis (PCA). The principal
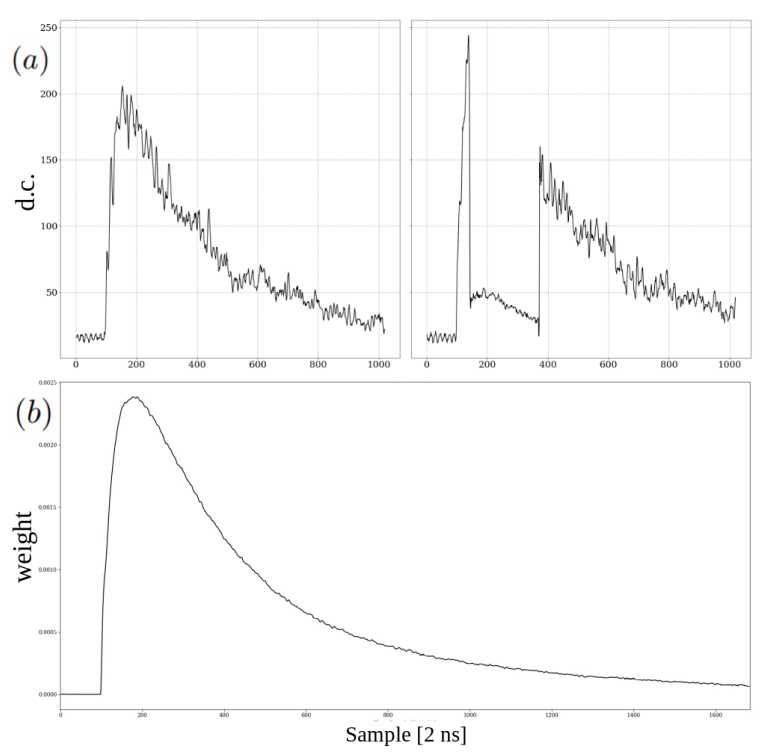

Figure 4: (a) A typical non-switched scintillation pulse recorded by the FADC system (le ft) and a pulse large enough to trigger the gain-switching (right). (b) The pulse-profile derived from non-switching events using PCA. This profile was used to estimate the switched pulse fraction. Adapted from [2]. component responsible for variability in the waveform data was the scintillation pulse itself (Fig. $4 \mathrm{~b}$ ), with higher components showing additional effects like prompt pulses from direct ionization in the WLS fibers. This putative pulse profile was used to calculate the fractional missing area in the integrated gain-switched trace.

To characterize the ICC prototype response to the fragmented beam, events were compared with the HNX silicon strip detector (SSD) charge measurements. In the absence of a shared event number, events between the two experiments were correlated based on the software event time 
stamps in each experiment. The time differences between inter-spill muons were used to calculate the cross-correlation timing offset between the two data sets and derive the ICC per-species charge response. The PMT capturing the relatively large, integrated signal from the bottom side of the CsI:Na crystal provided an unambiguous, independent signal that proved key to identifying timecorrelated events. The signal also provided a means to apply consistency cuts to the data to elminate events showing evidence for fragmentation or knock-on electrons produced in the detector volume, between the SSD and ICC detector. To compensate for any remaining miscorrelation in the WLS fiber events due to the relatively large uncorrelated dead time in the FADC system and the inherent timing jitter in the time stamping, a neighbor selection algorithm was run using the PMT-identified events. To further aid in matching the relatively sparse (higher dead-time) APT event data to the nearby HNX events, loose cuts on the PMT charge measurements were used to further remove ambiguities and identify the corresponding events.

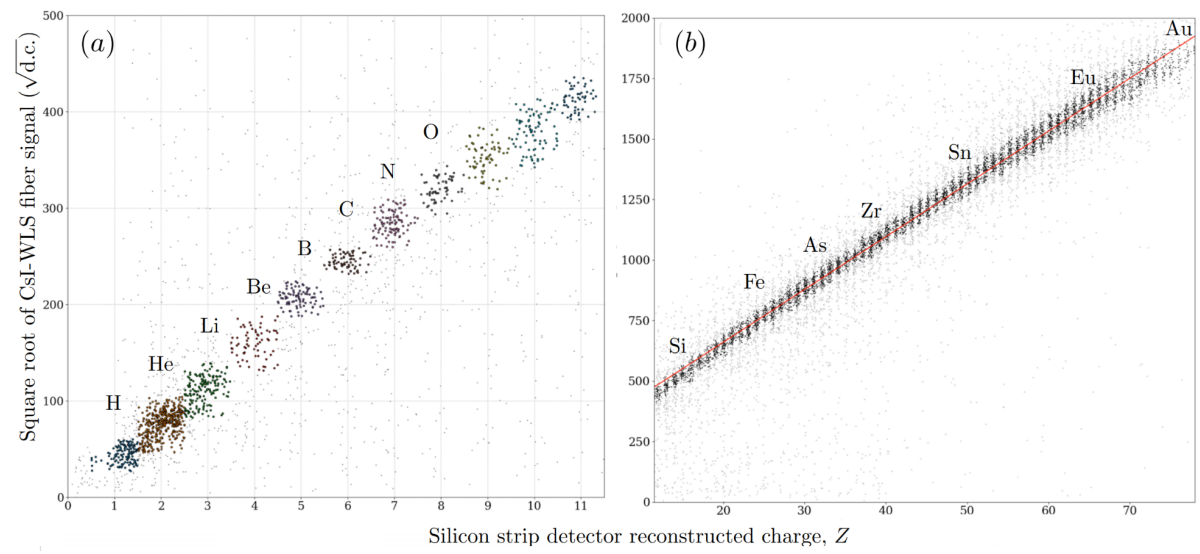

Figure 5: (a) The APT detector response vs. the HNX silicon-strip-determined atomic number for nuclei that did not trigger the VERITAS FADC gain switching. (b) The detector response where gain-switching took place. Adapted from [2].

Figure 5 shows the APT per-species charge response compared with the HNX silicon strip detector charge measurement. Due to the missing waveform data in the HILO gain-switched traces two regimes exist in the data: In the light nuclei regime $(Z<12)$ charge resolution is tight and distinct charge species can be identified. In the heavy nuclei regime $(Z \geq 12)$, the imperfect nature of the HILO gain correction leads to degraded charge resolution as atomic number increases. Still, the detector response shows a linear correlation with the SSD response with no rollover up to the highest nuclei measured $(Z=82)$. While Silicon is known to provide good charge measurements for high- $Z$ nuclei, this is one of the first demonstrations that thin CsI:Na lacks significant saturation effects for very dense ionization from heavy nuclei, establishing it as a viable detector material for ultra-heavy CR measurements with APT.

\section{APTlite}

About a year after the CERN beam test, a second generation detector, designated APTlite, was launched as a piggy-back experiment on the SuperTIGER-2.3 (ST) balloon flight which took place between December 12, 2019 and January 16, 2020. Since it was located under the center of the ST 
region, trajectory information measured in ST and charge determination could be used to form a roughly calibrated test beam for the APTlite instrument.

APTlite includes a number of incremental improvements over previous prototypes. Principally, the detector itself was a larger $150 \times 150 \times 5 \mathrm{~mm}$ CsI:Na crystal coupled to 128 red-green WLS fibers reading out both the $x$ - and $y$-planes. Additionally, the read-out electronics were replaced by 4 Target C/T5TEA boards. While these were custom boards, the ASICS were originally designed for use in CTA (like the VERITAS FADCs) as waveform digitizers designed for atmospheric Cherenkov measurements. The TC/T5TEA boards operate at $1 \mathrm{GSa} / \mathrm{s}$ with a $16 \mathrm{k}$ sample memory depth and independently configurable channels[4]. With modifications of the CTA firmware, the TARGET/T5TEA boards were able to read out $4 \mu$ s of waveform data. With nearly a factor-of-three increase in read-out depth compared to the CERN beam test, the TC/T5TEA modules allow for fitting over the entire scintillation pulse profile. The preamplifier and voltage control boards used in the APTlite experiment were essentially the same design as those used in the CERN beam test. The majority of data taken during APTlite's flight was taken with a SiPM voltage of $40.8 \mathrm{~V}$. A PC/104 computer stack within the detector box provides overall control, communication, triggering, and data storage for APTlite. Figure 6 shows the ICC prototype and the overall APTlite detector box.
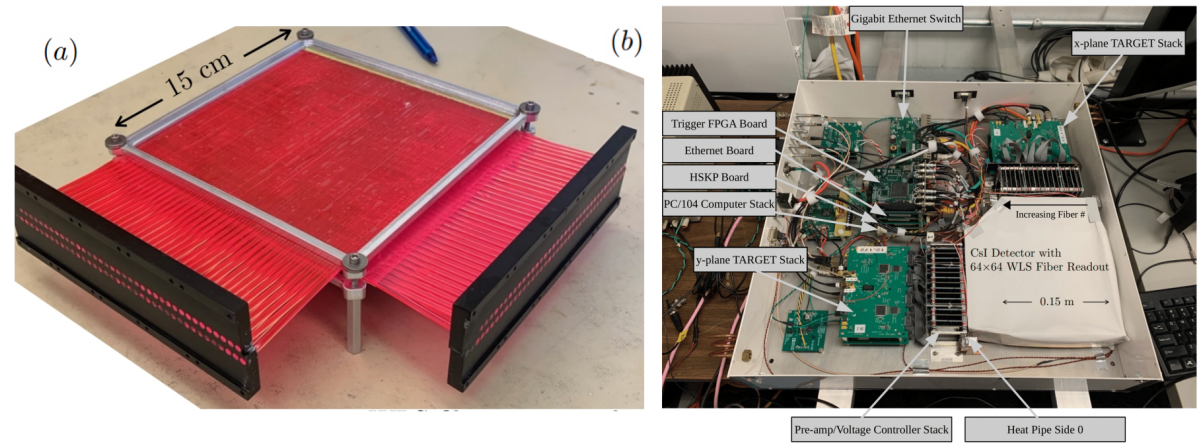

Figure 6: (a) The APTlite ICC prototype. (b) The APTlite instrument box before mounting onto the SuperTIGER gondola. Adapted from [2]

From SuperTIGER, APTlite received power, a trigger signal, and communication with the Iridium OpenPort device for ground communication. The three primary goals for the piggy-back flight were: (1) to advance the overall technical readiness of APT; (2) to demonstrate the ability to reconstruct charge for high- $A$ saturating cosmic rays; and (3) to determine the ability of APT to spatially resolve the positions of events within the detector plane.

\subsection{APTlite results}

Waveforms recorded by the TC/T5TEA electronics from the WLS fiber SiPMs were fit using an exponential model for the pulse profile, modified to capture the characteristic undershoot in the tail of the CsI:Na pulse in the TC waveforms. The functional form for the fit to the waveform data is a piece-wise function over three domains:

$$
y\left(x ; A, x_{0}, t_{r}, t_{f}, y_{0}, y_{1}, m\right)=\left\{\begin{array}{cc}
m x+y_{\text {start }} & 0 \leqslant x<x_{\text {edge }} \\
\frac{2 y_{r}}{\left(y_{r}+y_{l}\right)} A\left(1-e^{-\frac{x-x_{0}}{t_{r}}}\right)\left(e^{-\frac{x-x_{0}}{t_{f}}}\right)+y_{0} & x_{\text {edge }} \leqslant x<x_{\text {peak }} \\
\frac{2 y_{l}}{\left(y_{r}+y_{l}\right)} A\left(1-e^{-\frac{x-x_{0}}{t_{r}}}\right)\left(e^{-\frac{x-x_{0}}{t_{f}}}\right)+y_{1} & x_{\text {peak }} \leqslant x<x_{\text {end }} .
\end{array}\right.
$$


The first portion characterizes the pedestal region linearly from the beginning of the waveform to the rising edge of the pulse profile, $x_{\text {edge. }}$ The pulse itself is modeled as a smoothly connected two-sided exponential pulse defined by a rising time constant, $t_{r}$; a falling time constant, $t_{f}$; a time offset $x_{0}$; an overall amplitude A, and separate pedestal offsets, $y_{0}$ and $y_{1}$. The terms $y_{l}$ and $y_{r}$ ensure continuity. The model characterizes the profile well, as seen in Fig. 7.

For large amplitude cosmic ray events the preamp and TC electronics entered saturation. The model in Eq. 1 was fit to these events by first excluding the the portion of the waveform during saturation. Saturation was identified using a 50-sample sliding (box-car) standard deviation calculation and searching for regions where the standard deviation was too small or was monotonically increasing, an indicator that the electronics are entering saturation[5]. Because of the deep $4 \mu$ s read-out depth of the TARGET/T5TEA electronics, enough of the pulse remained to fit the waveforms. Figure 8 shows the fitting algorithm on both saturating and non-saturating

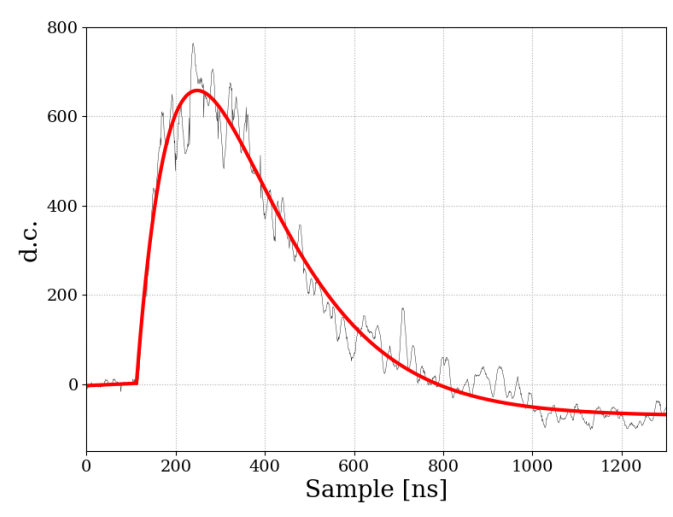

Figure 7: A non-saturating event for the APTlite balloon flight and the corresponding model fit. events for a putative silicon event.

Using the model-derived pulse heights for WLS fiber SiPM signals, cosmic rays passing through the APT detector could be localized from the light distribution measured either in the $x$ or $y$ fiber planes. A Gaussian fit was found to give a poor match to the measured spatial distribution of the scintillation event due to the highly peaked nature of the signal. Furthermore, fibers near the edge of the crystal recorded reflected light resulting in edge-effects that altered the signal's spatial profile. For this reason, a two-sided Voigt function (the convolution of a Gaussian and Lorentizan profile) was used as the spatial model:

$$
y\left(x ; A, x_{0}, \sigma_{1}, \gamma_{1}, y_{1}, \sigma_{2}, \gamma_{2}, y_{2}\right)= \begin{cases}A \frac{2 y_{r}}{\left(y_{r}+y_{l}\right)} V_{p}\left(x-x_{0} ; \sigma_{1}, \gamma_{1}\right)+y_{0} & x_{\text {start }} \leqslant x<x_{\text {peak }} \\ A \frac{2 y_{l}}{\left(y_{r}+y_{l}\right)} V_{p}\left(x-x_{0} ; \sigma_{2}, \gamma_{2}\right)+y_{1} & x_{\text {peak }} \leqslant x<x_{\text {end }}\end{cases}
$$

where A and $x_{0}$ are a common amplitude and channel offset, respectfully; $y_{0}$ and $y_{1}$ are separate pedestal offsets; the terms $y_{l}$ and $y_{r}$ ensure continuity; and $\sigma_{i}$ and $\gamma_{i}$ are the parameters of the Voigt function:

$$
V_{p}(x ; \sigma, \gamma)=\int_{-\mathrm{inf}}^{\mathrm{inf}} G\left(x^{\prime} ; \sigma\right) L\left(x-x^{\prime} ; \gamma\right) d x^{\prime} .
$$

For the $40.8 \mathrm{~V}$ data set, which comprised the bulk of the data collected during flight, the average full width at half maximum (FWHM) was $\approx 3$ fiber widths in both the $x$ - and $y$-planes. The centroid position can be determined more accurately, but depends on the details of the signal amplitude, edge effects and other corrections but is typically much less than a single fiber width. Figure 8 shows a typical event passing data quality cuts and its corresponding Voigt-fit using the WLS fiber pulse heights.

\section{Summary}

APT aims to be a true particle-astrophysics telescope, capable of multimessanger physics as both a gamma-ray and cosmic-ray instrument with incredibly broad spectral reach from $\mathrm{MeV}-\mathrm{TeV}$ 


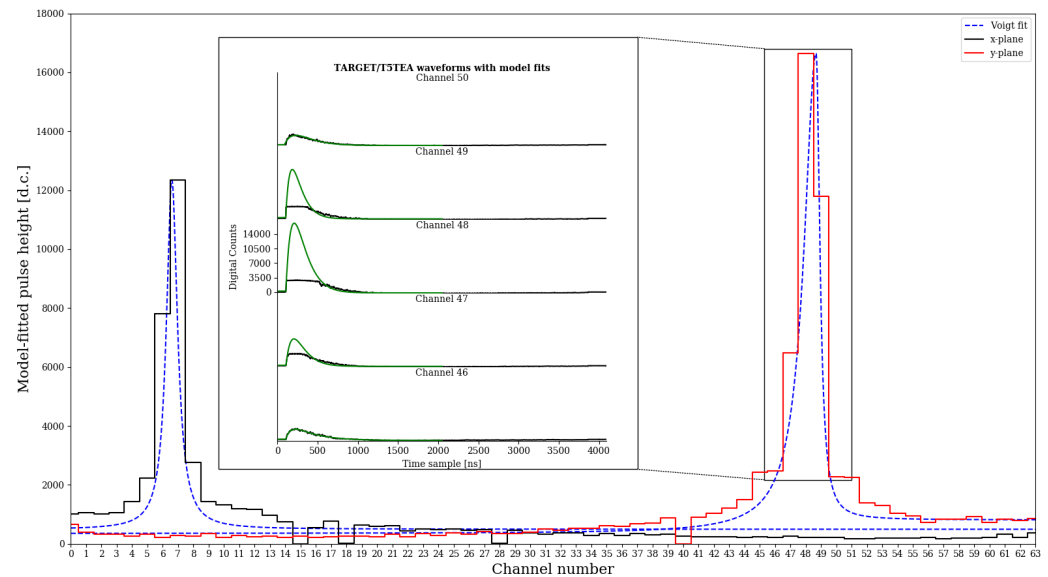

Figure 8: Silicon event reconstruction showing pulse profile fits to saturating and non-saturating channels (inset). A two-sided Voigt function fit to the spatial profile of the event (blue). Adapted from [2].

energies. This requires an instrument with large dynamic range, capable of both resolving single photo-electrons in the low-energy regime and reconstructing saturating events at the high-energy extreme. Current evaluation of the imaging CsI calorimeter in a beam test and aboard an Antarctic balloon shows its potential as a cosmic-ray instrument. Results form the 2018 CERN beam test show distinct charge identification for non-electronics-limited nuclei $(Z<12)$ and a linear detector response over a range of nuclear charges (up to lead) even in a regime of significant electronics saturation. The 2019 Antarctic balloon flight on board SuperTIGER shows event reconstruction from the long-tail decay of CsI:Na enabled by the deep memory available to the TARGET family of ASICs. Furthermore, spatial localization within the fiber plane is fine enough ( $<$ a fiber width) within the $150 \times 150 \times 5 \mathrm{~mm}$ tile to allow for trajectory reconstruction of incident particles. Further evaluation in beam tests with a 4 layer tracker and calorimeter and a dedicated balloon instrument (ADAPT[1]) will provide improved constraints on instrument performance and directly demonstrate the gamma-ray performance of the instrument.

\section{References}

[1] J. Buckley, The Advanced Particle-astrophysics Telescope (APT) Project Status, in Proceedings of 37th International Cosmic Ray Conference - PoS(ICRC2021), vol. 395, p. 655, 2021, DOI.

[2] Z. Hughes, Toward an Understanding of High-mass Gamma-ray Binaries: An Investigation Using Current Observatories and the Development of a Future GeV Instrument, Ph.D. thesis, Washington University in St. Louis, Jan, 2021. https: //www . proquest . com/docview/28264702.

[3] J.F. Krizmanic and et al., HNX/SuperTIGER Silicon Strip Detector Response to Nuclei in Lead Primary and Fragmented Test Beams, in Proceedings of 36th International Cosmic Ray Conference - PoS (ICRC2019), vol. 358, p. 94, 2019.

[4] S. Funk, D. Jankowsky, H. Katagiri, M. Kraus, A. Okumura, H. Schoorlemmer et al., Target: A digitizing and trigger asic for the cherenkov telescope array, AIP Conference Proceedings 1792 (2017) 080012 [https://aip.scitation.org/doi/pdf/10.1063/1.4969033].

[5] J. Schafer, Temperature dependent aspects of the compact high energy camera (chec-s) front-end electronic (fee) calibration, Master's thesis, University of Erlangen-Nuremberg, Apr, 2019, [https://ecap.nat.fau.de/wp-content/uploads/2019/04/2019_Schaefer_Master.pdf]. 


\section{Full Authors List: APT Collaboration}

S. Alnussirat ${ }^{1}$, C. Altomare ${ }^{2}$, R. G. Bose ${ }^{3}$, D. Braun ${ }^{3}$, J. H. Buckley ${ }^{3}$, J. D. Buhler ${ }^{4}$, E. Burns ${ }^{1}$, R. D. Chamberlain ${ }^{4}$, W. Chen ${ }^{5}$, M. L. Cherry ${ }^{1}$, L. Di Venere ${ }^{6,2}$, J. Dumonthier ${ }^{7}$, M. Errando ${ }^{3}$, S. Funk ${ }^{8}$, F. Giordano ${ }^{6,2}$, J. Hoffman ${ }^{3}$, Z. Hughes $^{3}$, D. J. Huth ${ }^{3}$, P. L. Kelly ${ }^{5}$, J. F. Krizmanic ${ }^{9,10,11}$, M. Kuwahara ${ }^{12}$, F. Licciulli ${ }^{2}$, G. Liu ${ }^{13}$, M. N. Mazziotta ${ }^{2}$, J. G. Mitchell ${ }^{14,15}$, J. W. Mitchell ${ }^{9}$, G. A. de Nolfo ${ }^{15}$, R. Paoletti ${ }^{16}$, R. Pillera ${ }^{17,2}$, B. F. Rauch ${ }^{3}$, D. Serini ${ }^{2}$, G. Simburger ${ }^{3}$, M. Sudvarg ${ }^{4}$, G. Suarez ${ }^{7}$, T. Tatoli ${ }^{15,18}$, G. S. Varner ${ }^{12}$, E. Wulf ${ }^{19}$, A. Zink ${ }^{8}$, W. V. Zober ${ }^{3}$

${ }^{1}$ Department of Physics and Astronomy, Louisiana State University, Baton Rouge, Louisiana 70803, USA, ${ }^{2}$ Istituto Nazionale di Fisica Nucleare, Sezione di Bari, I-70126 Bari, Italy, ${ }^{3}$ Department of Physics and McDonnell Center for the Space Sciences, Washington University, St. Louis, MO 63130, USA, ${ }^{4}$ Department of Computer Science \& Engineering, Washington University, St. Louis, MO 63130, USA, ${ }^{5}$ Department of Physics and Astronomy, University of Minnesota, Minneapolis, MN 55455, USA, ${ }^{6}$ Dipartimento di Fisica "M. Merlin" dell'Università e del Politecnico di Bari, I-70126 Bari, Italy, ${ }^{7}$ NASA Goddard Space Flight Center, Greenbelt, MD 20771, USA, ${ }^{8}$ Friedrich-Alexander-Universität Erlangen-Nürnberg, Erlangen Centre for Astroparticle Physics, D-91058 Erlangen, Germany, ${ }^{9}$ Center for Space Sciences and Technology, University of Maryland Baltimore County, Baltimore, MD 21250, USA, ${ }^{10}$ Astroparticle Physics Laboratory, NASA/GSFC, Greenbelt, MD 20771, USA, ${ }^{11}$ Center for Research and Exploration in Space Sciences and Technology, NASA/GSFC, Greenbelt, MD 20771, USA, ${ }^{12}$ Department of Engineering, University of Hawai ‘i at Mānoa, Honolulu, HI 96822, USA, ${ }^{13}$ Department of Physics and Astronomy, University of Hawai ' $i$ at Mānoa, Honolulu, HI 96822, USA, ${ }^{14}$ Department of Physics, The George Washington University, Washington, DC 20052, USA, ${ }^{15}$ Heliospheric Physics Laboratory, NASA/GSFC, Greenbelt, MD 20771, USA, ${ }^{16}$ Università di Siena and INFN Pisa, I-53100 Siena, Italy, ${ }^{17}$ Politecnico di Bari, Department of Mechanics, Mathematics and Management, via Orabona, 4, I-70125 Bari, Italy, ${ }^{18}$ Department of Physics, Catholic University of America, Washington, DC 20064, USA, ${ }^{19}$ Naval Research Laboratory, Washington, DC 20375, USA 